\title{
ON THE COHOMOLOGICAL DIMENSION OF THE LOCALIZATION FUNCTOR
}

\author{
HENRYK HECHT AND DRAGAN MILIČIĆ
}

(Communicated by Jonathan M. Rosenberg)

\begin{abstract}
The left cohomological dimension of the localization functor is infinite for singular infinitesimal characters.
\end{abstract}

Let $\mathfrak{g}$ be a complex semisimple Lie algebra and $X$ the flag variety of $\mathfrak{g}$, i.e. the variety of all Borel subalgebras in $\mathfrak{g}$. Let $\mathfrak{h}$ be the (abstract) Cartan algebra of $\mathfrak{g}, \Sigma$ the root system in $\mathfrak{h}^{*}$ and $\Sigma^{+}$the set of positive roots determined by the condition that the homogeneous line bundles $\mathscr{O}(-\mu)$ on $X$ corresponding to dominant weights $\mu$ are positive. Denote by $W$ the Weyl group of $\Sigma$. By a well-known result of Harish-Chandra the center $\mathscr{Z}(\mathfrak{g})$ of the enveloping algebra $\mathscr{U}(\mathfrak{g})$ is isomorphic to the Weyl group invariants $I(\mathfrak{h})$ in the symmetric algebra $S(\mathfrak{h})$. Therefore, the space of all maximal ideals in $\mathscr{Z}(\mathfrak{g})$ can be identified with the $W$-orbits in $\mathfrak{h}^{*}$. Let $\theta$ be such an orbit in $\mathfrak{h}^{*}$, and denote by $J_{\theta}$ the corresponding maximal ideal in $\mathscr{Z}(\mathfrak{g})$. Put $\mathscr{U}_{\theta}=\mathscr{U}(\mathfrak{g}) / J_{\theta} \mathscr{U}(\mathfrak{g})$. Denote by $\mathscr{M}\left(\mathscr{U}_{\theta}\right)$ the category of $\mathscr{U}_{\theta}$-modules.

For any $\lambda \in \mathfrak{h}^{*}$, A. Beilinson and J. Bernstein defined a twisted sheaf of differential operators $\mathscr{D}_{\lambda}$ on $X$ with the property that $\Gamma\left(X, \mathscr{D}_{\lambda}\right)=\mathscr{U}_{\theta}$ (compare [1], [6]). Denote by $\hat{\mathscr{M}}_{\text {qc }}\left(\mathscr{D}_{\lambda}\right)$ the category of quasicoherent $\mathscr{D}_{\lambda}$-modules on $X$. They also defined the localization functor $\Delta_{\lambda}$ from $\mathscr{M}\left(\mathscr{U}_{\theta}\right)$ into $\mathscr{M}_{\mathrm{qc}}\left(\mathscr{D}_{\lambda}\right)$ by the formula

$$
\Delta_{\lambda}(V)=\mathscr{D}_{\lambda} \otimes_{\mathscr{U}_{\theta}} V
$$

for a $\mathscr{U}_{\theta}$-module $V$.

Let $Q(\Sigma)$ be the root lattice in $\mathfrak{h}^{*}$. For any $\lambda \in \mathfrak{h}^{*}$, we denote by $W_{\lambda}$ the subgroup of the Weyl group $W$ given by $W_{\lambda}=\{w \in W \mid w \lambda-\lambda \in Q(\Sigma)\}$. Let $\Sigma^{\vee}$ be the root system in $\mathfrak{h}$ dual to $\Sigma$; and for any $\alpha \in \dot{\Sigma}$, we denote by $\alpha^{\vee} \in \Sigma^{\vee}$ the dual root of $\alpha$. Then, it is well-known that $W_{\lambda}$ is the Weyl group of the root system $\Sigma_{\lambda}=\left\{\alpha \in \Sigma \mid \alpha^{\vee}(\lambda) \in \mathbf{Z}\right\}$. We define an order on $\Sigma_{\lambda}$ by putting $\Sigma_{\lambda}^{+}=\Sigma^{+} \cap \Sigma_{\lambda}$. This defines a set of simple roots $\Pi_{\lambda}$ of $\Sigma_{\lambda}$, and the corresponding set of simple reflections $S_{\lambda}$. Let $l_{\lambda}$ be the length function on $\left(W_{\lambda}, S_{\lambda}\right)$.

Received by the editors January 23, 1989.

1980 Mathematics Subject Classification (1985 Revision). Primary 22E47.

Supported in part by NSF Grant DMS 88-02827. 
We say that $\lambda \in \mathfrak{h}^{*}$ is regular if $\alpha^{\vee}(\lambda)$ is different from zero for any $\alpha \in \Sigma$ and that $\lambda$ is antidominant if $\alpha^{\vee}(\lambda)$ is not a strictly positive integer for any $\alpha \in \Sigma^{+}$. We put $n(\lambda)=\min \left\{l_{\lambda}(w) \mid w \in W_{\lambda}, w \lambda\right.$ is antidominant $\}$. Beilinson and Bernstein proved that, for regular $\lambda$, the left cohomological dimension of the localization functor is $\leq n(\lambda)([2],[8])$. In this note we prove the following complementary result.

Theorem. Let $\lambda \in \mathfrak{h}^{*}$ be singular. Then the left cohomological dimension of the localization functor $\Delta_{\lambda}$ is infinite.

Using the fact that the localization functor is an equivalence of the category $\mathscr{M}\left(\mathscr{U}_{\theta}\right)$ with the category $\mathscr{M}_{\mathrm{qc}}\left(\mathscr{D}_{\lambda}\right)$ for regular antidominant $\lambda$, Beilinson and Bernstein also proved that the homological dimension of $\mathscr{U}_{\theta}$ is $\leq \frac{1}{2}\left(\operatorname{Card}(\Sigma)+\operatorname{Card}\left(\Sigma_{\lambda}\right)\right)$ if $\theta=W \cdot \lambda$ consists of regular elements (unpublished, compare [8]). On the contrary, our result immediately implies the following consequence.

Corollary. If $\theta$ consists of singular elements in $\mathfrak{h}^{*}$, the homological dimension of $\mathscr{U}_{\theta}$ is infinite.

This fact was observed earlier by A. Joseph and J. T. Stafford ([7, 4.20]). Our argument shows that this is a simple consequence of the analogous behavior of homological dimension for local rings.

Proof of the theorem. Let $x$ be a point in $X$ and denote by $\mathfrak{b}_{x}$ the corresponding Borel subalgebra of $\mathfrak{g}$. Let $\mathfrak{n}_{x}=\left[\mathfrak{b}_{x}, \mathfrak{b}_{x}\right]$ be its nilpotent radical. Let $\mathfrak{h}_{x}=\mathfrak{b}_{x} / \mathfrak{n}_{x}$. Then $\mathfrak{h}_{x}$ is canonically isomorphic to $\mathfrak{h}$ [6]. Let $\mathfrak{c}$ be a Cartan subalgebra of $\mathfrak{g}$ contained in $\mathfrak{b}_{x}$. Then the composition of the projection $\mathfrak{c} \rightarrow \mathfrak{h}_{x}$ with this map gives an isomorphism of $\mathfrak{c}$ onto $\mathfrak{h}$. The inverse map is called a specialization at $x$. For a $\mathscr{U}(\mathfrak{g})$-module $V$, we put

$$
V_{\mathfrak{n}_{x}}=V / \mathfrak{n}_{x} V=\mathbf{C} \otimes_{\mathscr{U}_{\left(\mathfrak{n}_{x}\right)}} V,
$$

where we view $\mathbf{C}$ as a module with the trivial action of $\mathfrak{b}_{x}$. It has a natural structure of an $\mathfrak{h}_{x}$-module. Therefore, we can view it as an $\mathfrak{h}$-module. It follows that $V \rightarrow V_{n_{x}}$ is a right exact covariant functor from the category of $\mathscr{U}(\mathfrak{g})$-modules into the category of $\mathscr{U}(\mathfrak{h})$-modules. If we compose it with the forgetful functor into the category of vector spaces, we get the functor $H_{0}\left(\mathfrak{n}_{x},-\right)$ of zeroth $\mathfrak{n}_{x}$-homology. By the Poincaré-Birkhoff-Witt theorem, free $\mathscr{U}(\mathfrak{g})$ modules are also $\mathscr{U}\left(n_{x}\right)$-free, what implies the equality for the left derived functors. Therefore, with some abuse of language, we view the $(-p)^{\text {th }}$ left derived functor of $V \rightarrow V_{\mathfrak{n}_{x}}$ as the $p^{\text {th }} \mathfrak{n}_{x}$-homology functor $H_{p}\left(\mathfrak{n}_{x},-\right)=$ $\operatorname{Tor}_{p}^{\mathscr{U}\left(\mathbf{n}_{x}\right)}(\mathbf{C},-)$.

We need a technical result, which must be well known, but we were unable to find a reference.

1. Lemma. $\mathscr{U}_{\theta}$ is free as $\mathscr{U}\left(\mathfrak{n}_{x}\right)$-module. 
Proof. Let $\mathfrak{c}$ be a Cartan subalgebra of $\mathfrak{g}$ contained in $\mathfrak{b}_{x}$. This determines a specialization of $\mathfrak{h}$ to $\mathfrak{c}$ and a nilpotent subalgebra $\overline{\mathfrak{n}}$ opposite to $\mathfrak{n}_{x}$. Then we have $\mathfrak{g}=\mathfrak{n}_{x} \oplus \mathfrak{c} \oplus \overline{\mathfrak{n}}$ and $\mathscr{U}(\mathfrak{g})=\mathscr{U}\left(\mathfrak{n}_{x}\right) \otimes_{\mathbf{C}} \mathscr{U}(\mathfrak{c}) \otimes_{\mathbf{C}} \mathscr{U}(\overline{\mathfrak{n}})$ as a left $\mathscr{U}\left(\mathfrak{n}_{x}\right)$-module for left multiplication. Let $\mathrm{F}_{p} \mathscr{U}(\mathfrak{c}), p \in \mathbf{Z}_{+}$, be the degree filtration of $\mathscr{U}(\mathfrak{c})$. Then we define a filtration $\mathrm{F}_{p} \mathscr{U}(\mathfrak{g}), p \in \mathbf{Z}_{+}$, of $\mathscr{U}(\mathfrak{g})$ via

$$
\mathrm{F}_{p} \mathscr{U}(\mathfrak{g})=\mathscr{U}\left(\mathfrak{n}_{x}\right) \otimes_{\mathbf{C}} \mathrm{F}_{p} \mathscr{U}(\mathfrak{c}) \otimes_{\mathbf{C}} \mathscr{U}(\overline{\mathfrak{n}}) .
$$

This is clearly a $\mathscr{U}\left(\mathfrak{n}_{x}\right)$-module filtration. The corresponding graded module is

$$
\operatorname{Gr} \mathscr{U}(\mathfrak{g})=\mathscr{U}\left(\mathfrak{n}_{x}\right) \otimes_{\mathbf{C}} S(\mathfrak{c}) \otimes_{\mathbf{C}} \mathscr{U}(\overline{\mathfrak{n}}) .
$$

This filtration induces a filtration on the submodule $J_{\theta} \mathscr{U}(\mathfrak{g})$ and the quotient module $\mathscr{U}_{\theta}$. The Harish-Chandra homomorphism $\gamma: \mathscr{Z}(\mathfrak{g}) \rightarrow \mathscr{U}(\mathfrak{h})$ is compatible with the degree filtrations and the homomorphism $\mathrm{Gr} \gamma$ is an isomorphism of $\operatorname{Gr} \mathscr{Z}(\mathfrak{g})$ onto the subalgebra $I(\mathfrak{h})$ of all $W$-invariants in $S(\mathfrak{h})$ ([4, Ch. VIII, $\S 8$, no. 5]). Denote by $I_{+}(\mathfrak{h})$ the homogeneous ideal spanned by the elements of strictly positive degree in $I(\mathfrak{h})$. Then

$$
\operatorname{Gr} J_{\theta} \mathscr{U}(\mathfrak{g})=\mathscr{U}\left(\mathfrak{n}_{x}\right) \otimes_{\mathbf{C}} I_{+}(\mathfrak{c}) S(\mathfrak{c}) \otimes_{\mathbf{C}} \mathscr{U}(\overline{\mathfrak{n}}) .
$$

It follows that

$$
\begin{aligned}
\operatorname{Gr} \mathscr{U}_{\theta} & =(\operatorname{Gr} \mathscr{U}(\mathfrak{g})) /\left(\operatorname{Gr} J_{\theta} \mathscr{U}(\mathfrak{g})\right) \\
& =\left(\mathscr{U}\left(\mathfrak{n}_{x}\right) \otimes_{\mathbf{C}} S(\mathfrak{c}) \otimes_{\mathbf{C}} \mathscr{U}(\overline{\mathfrak{n}})\right) /\left(\mathscr{U}\left(\mathfrak{n}_{x}\right) \otimes_{\mathbf{C}} I_{+}(\mathfrak{c}) S(\mathfrak{c}) \otimes_{\mathbf{C}} \mathscr{U}(\overline{\mathfrak{n}})\right) \\
& =\mathscr{U}\left(\mathfrak{n}_{x}\right) \otimes_{\mathbf{C}}\left(S(\mathfrak{c}) /\left(I_{+}(\mathfrak{c}) S(\mathfrak{c})\right)\right) \otimes_{\mathbf{C}} \mathscr{U}(\overline{\mathfrak{n}}),
\end{aligned}
$$

i.e. it is a free $\mathscr{U}\left(\mathfrak{n}_{x}\right)$-module. Moreover, by ([4, Ch. V, $\S 5$, no. 2, Th. 1]) we know that the dimension of the complex vector space $S(\mathfrak{h}) /\left(I_{+}(\mathfrak{h}) S(\mathfrak{h})\right)$ is Card $W$. It follows that $\mathscr{U}_{\theta}$ has a finite filtration by $\mathscr{U}\left(\mathfrak{n}_{x}\right)$-submodules such that $\mathrm{Gr} \mathscr{U}_{\theta}$ is a free $\mathscr{U}\left(\mathfrak{n}_{x}\right)$-module. By induction in length, this implies that $\mathscr{U}_{\theta}$ is a free $\mathscr{U}\left(\mathfrak{n}_{x}\right)$-module.

Let $\rho$ be the half-sum of roots in $\Sigma^{+}$. Denote by $\varphi: \mathscr{U}(\mathfrak{h}) \rightarrow \mathscr{U}(\mathfrak{h})$ the automorphism given by $\varphi(\xi)=\xi+\rho(\xi)$ for $\xi \in \mathfrak{h}$. Then, $\varphi(\gamma(\mathscr{Z}(\mathfrak{g})))$ is the algebra of $W$-invariants in $\mathscr{U}(\mathfrak{h})$. In addition, as we remarked in the preceding proof, the dimension of the vector space $\mathscr{U}(\mathfrak{h}) / \varphi\left(\gamma\left(J_{\theta}\right)\right) \mathscr{U}(\mathfrak{h})$ is equal to Card $W$. This implies that $V_{\theta}=\mathscr{U}(\mathfrak{h}) / \gamma\left(J_{\theta}\right) \mathscr{U}(\mathfrak{h})$ is an $\mathscr{U}(\mathfrak{h})$-module of dimension $\operatorname{dim}_{\mathbf{C}} V_{\theta}=$ Card $W$.

For $\mu \in \mathfrak{h}^{*}$, we denote by $I_{\mu}$ the corresponding maximal ideal in $\mathscr{U}(\mathfrak{h})$.

2. Lemma. Let $\lambda \in \mathfrak{h}^{*}$ and $\theta=W \cdot \lambda$. Then:

(i) $V_{\theta}$ is a $\mathscr{U}(\mathfrak{h})$-module of dimension $\operatorname{dim}_{\mathrm{C}} V_{\theta}=\mathrm{Card} W$;

(ii) the characteristic polynomial of the action of $\xi \in \mathfrak{h}$ on $V_{\theta}$ is

$$
P(\xi)=\prod_{w \in W}(\xi-(w \lambda+\rho)(\xi)) ;
$$

(iii) $H_{0}\left(\mathfrak{n}_{x}, \mathscr{U}_{\theta}\right)$ is a direct sum of countably many copies of $V_{\theta}$. 
Proof. We already established (i). Clearly, $I_{\mu} \supset \varphi\left(\gamma\left(J_{\theta}\right)\right) \mathscr{U}(\mathfrak{h})$ is equivalent to $\mu=w \lambda$ for some $w \in W$. Hence the linear transformation of $\mathscr{U}(\mathfrak{h}) / \varphi\left(\gamma\left(J_{\theta}\right)\right) \mathscr{U}(\mathfrak{h})$ induced by multiplication by $\xi$ has eigenvalues $(w \lambda)(\xi)$, $w \in W$, and by symmetry they all have the same multiplicity. This in turn implies that

$$
\varphi(P(\xi))=\prod_{w \in W} \varphi(\xi-(w \lambda+\rho)(\xi))=\prod_{w \in W}(\xi-(w \lambda)(\xi))
$$

is the characteristic polynomial for the action of $\xi$ on $\mathscr{U}(\mathfrak{h}) / \varphi\left(\gamma\left(J_{\theta}\right)\right) \mathscr{U}(\mathfrak{h})$. This proves (ii).

(iii) As in the proof of 1 , we fix a specialization $\mathfrak{c}$ of $\mathfrak{h}$ and choose a nilpotent subalgebra $\overline{\mathfrak{n}}$ opposite to $\mathfrak{n}_{x}$. By the Poincaré-Birkhoff-Witt theorem, it follows that as a vector space $\mathscr{U}(\mathfrak{g})=\mathscr{U}\left(\mathfrak{n}_{x}\right) \otimes_{\mathbf{C}} \mathscr{U}(\mathfrak{c}) \otimes_{\mathbf{C}} \mathscr{U}(\overline{\mathfrak{n}})$. Moreover,

$$
H_{0}\left(\mathfrak{n}_{x}, \mathscr{U}_{\theta}\right)=\mathscr{U}(\mathfrak{g}) /\left(J_{\theta} \mathscr{U}(\mathfrak{g})+\mathfrak{n}_{x} \mathscr{U}(\mathfrak{g})\right) .
$$

Denote by $\gamma_{x}: \mathscr{Z}(\mathfrak{g}) \rightarrow \mathscr{U}(\mathfrak{c})$ the composition of the specialization map with the Harish-Chandra homomorphism $\gamma$. Then

$$
J_{\theta} \mathscr{U}(\mathfrak{g})+\mathfrak{n}_{x} \mathscr{U}(\mathfrak{g})=J_{\theta} \mathscr{U}(\mathfrak{c}) \mathscr{U}(\overline{\mathfrak{n}})+\mathfrak{n}_{x} \mathscr{U}(\mathfrak{g})=\gamma_{x}\left(J_{\theta}\right) \mathscr{U}(\mathfrak{c}) \mathscr{U}(\overline{\mathfrak{n}})+\mathfrak{n}_{x} \mathscr{U}(\mathfrak{g}),
$$

which implies that under the above isomorphism

$$
J_{\theta} \mathscr{U}(\mathfrak{g})+\mathfrak{n}_{x} \mathscr{U}(\mathfrak{g})=\left(\mathbf{C} \otimes_{\mathbf{C}} \gamma_{x}\left(J_{\theta}\right) \mathscr{U}(\mathfrak{c}) \otimes_{\mathbf{C}} \mathscr{U}(\overline{\mathfrak{n}})\right) \oplus\left(\mathfrak{n}_{x} \mathscr{U}\left(\mathfrak{n}_{x}\right) \otimes_{\mathbf{C}} \mathscr{U}(\mathfrak{c}) \otimes_{\mathbf{C}} \mathscr{U}(\overline{\mathfrak{n}})\right) .
$$

This yields

$$
H_{0}\left(\mathfrak{n}_{x}, \mathscr{U}_{\theta}\right)=\mathscr{U}(\mathfrak{c}) /\left(\gamma_{x}\left(J_{\theta}\right) \mathscr{U}(\mathfrak{c})\right) \otimes_{\mathbf{C}} \mathscr{U}(\overline{\mathfrak{n}})=V_{\theta} \otimes_{\mathbf{C}} \mathscr{U}(\overline{\mathfrak{n}})
$$

and the action of $\mathfrak{h}$ is given by multiplication in the first factor.

Therefore, maximal ideals in the ring $V_{\theta}$ are the images of the maximal ideals $I_{w \lambda+\rho}, w \in W$, in $\mathscr{U}(\mathfrak{h})$, under the quotient map $\mathscr{U}(\mathfrak{h}) \rightarrow V_{\theta}$.

Let $W(\lambda)$ be the stabilizer of $\lambda$ in $W$. Denote by $R_{w \lambda}$ the localization of $V_{\theta}$ at $I_{w \lambda+\rho}$. Then, by ([3, Ch. IV, $\S 2$, no. 5, Cor. 1 of Prop. 9]), we have

$$
V_{\theta}=\prod_{w \in W / W(\lambda)} R_{w \lambda} \text {. }
$$

Since the local rings $R_{w \lambda}$ are finite-dimensional, they are regular if and only if $\operatorname{dim}_{\mathrm{C}} R_{w \lambda}=1$. Because of the Weyl group symmetry these rings are isomorphic, hence $\operatorname{dim}_{\mathrm{C}} R_{w \lambda}=\operatorname{Card} W(\lambda)$ for any $w \in W$. This finally leads to the following critical observation.

3. Corollary. The following conditions are equivalent:

(i) $\lambda$ is regular;

(ii) the rings $R_{w \lambda}, w \in W$, are regular local rings.

By 1, we can calculate $\mathfrak{n}_{x}$-homology of $V$ using a left resolution of $V$ by free $\mathscr{U}_{\theta}$-modules. Therefore, we can view $H_{p}\left(\mathfrak{n}_{x}, V\right)$ as $V_{\theta}$-modules. Also, for any $\lambda \in \theta, \mathbf{C}_{\lambda+\rho}=\mathscr{U}(\mathfrak{h}) / I_{\lambda+\rho}$ is a $V_{\theta}$-module. 
For any $\mathscr{O}_{X}$-module $\mathscr{F}$ on $X$ we denote by $T_{x}(\mathscr{F})$ its geometric fibre at $x$, i.e. $T_{x}(\mathscr{F})=\mathrm{C} \otimes_{\mathscr{O}_{x}} \mathscr{F}_{x}$, where $\mathscr{O}_{x}$ is the local ring of $X$ at $x$. Since $X$ is a smooth algebraic variety, $\mathscr{O}_{x}$ is a regular local ring. Hence, the left cohomological dimension of the right exact functor $T_{x}$ is $\leq \operatorname{dim} X$.

For any abelian category $\mathscr{A}$, denote by $D^{-}(\mathscr{A})$ the derived category of $\mathscr{A}$ complexes bounded from above, and by $D$ the natural imbedding of $\mathscr{A}$ into $D^{-}(\mathscr{A})$ which maps an object $V$ of $\mathscr{A}$ into the complex $D(V)$ such that $D(V)^{p}=0$ for $p \neq 0$ and $D(V)^{0}=V$.

Since the localization functor $\Delta_{\lambda}$ is right exact, it defines the left derived functor $L \Delta_{\lambda}$ from $D^{-}\left(\mathscr{U}_{\theta}\right)$ into $D^{-}\left(\mathscr{D}_{\lambda}\right)$. Analogously, $T_{x}$ defines the left derived functor $L T_{x}$ from $D^{-}\left(\mathscr{D}_{\lambda}\right)$ into the derived category $D^{-}(\mathbf{C})$ of complexes of complex vector spaces bounded from above.

4. Proposition. Let $\lambda \in \mathfrak{h}^{*}, \theta=W \cdot \lambda$ and $x \in X$. Then the functors $L T_{x} \circ L \Delta_{\lambda}$ and $D\left(\mathbf{C}_{\lambda+\rho}\right) \stackrel{L}{\otimes}_{V_{\theta}}\left(D(\mathbf{C}) \stackrel{L}{\otimes}_{\mathscr{U}\left(\mathfrak{n}_{x}\right)}-\right)$ from $D^{-}\left(\mathscr{U}_{\theta}\right)$ into $D^{-}(\mathbf{C})$ are isomorphic.

Proof. By 1, we know that $\mathscr{U}_{\theta}$ is acyclic for the functor $H_{0}\left(\mathfrak{n}_{x},-\right)=\mathbf{C} \otimes_{\mathscr{U}\left(\mathfrak{n}_{x}\right)}-$. By 2, we also know that $\mathbf{C} \otimes_{\mathscr{U}\left(\mathrm{n}_{x}\right)} \mathscr{U}_{\theta}$ is acyclic for the functor $\mathbf{C}_{\lambda+\rho} \otimes_{V_{\theta}}-$. Let $F^{\cdot}$ be a complex isomorphic to $V^{\cdot}$ consisting of free $\mathscr{U}_{\theta}$-modules. Then, since the functors commute with infinite direct sums, we get

$$
D\left(\mathbf{C}_{\lambda+\rho}\right) \stackrel{L}{\otimes_{V_{\theta}}}\left(D(\mathbf{C}) \stackrel{L}{\otimes}_{\mathscr{U}\left(\mathfrak{n}_{x}\right)} V^{*}\right)=\mathbf{C}_{\lambda+\rho} \otimes_{V_{\theta}}\left(\mathbf{C} \otimes_{\mathscr{U}\left(\mathfrak{n}_{x}\right)} F^{*}\right) .
$$

On the other hand, the localization $\Delta_{\lambda}\left(\mathscr{U}_{\theta}\right)=\mathscr{D}_{\lambda}$ is a locally free $\mathscr{O}_{X}$-module, and therefore acyclic for $T_{x}$. This implies that

$$
L T_{x}\left(L \Delta_{\lambda}\left(V^{*}\right)\right)=T_{x}\left(\Delta_{\lambda}\left(F^{*}\right)\right) .
$$

Hence, to complete the proof it is enough to establish the following identity

$$
T_{x}\left(\Delta_{\lambda}\left(\mathscr{U}_{\theta}\right)\right)=\mathbf{C}_{\lambda+\rho} \otimes_{V_{\theta}}\left(\mathbf{C} \otimes_{\mathscr{U}\left(\mathbf{n}_{x}\right)} \mathscr{U}_{\theta}\right) .
$$

First, we have $T_{x}\left(\Delta_{\lambda}\left(\mathscr{U}_{\theta}\right)\right)=T_{x}\left(\mathscr{D}_{\lambda}\right)$. Moreover, from the construction of $\mathscr{D}_{\lambda}$ ([1], [6]) and the properties of the Harish-Chandra homomorphism, it follows that

$$
\begin{aligned}
T_{x}\left(\mathscr{D}_{\lambda}\right) & =\left(\mathscr{U}(\mathfrak{g}) / \mathfrak{n}_{x} \mathscr{U}(\mathfrak{g})\right) /\left(I_{\lambda+\rho}\left(\mathscr{U}(\mathfrak{g}) / \mathfrak{n}_{x} \mathscr{U}(\mathfrak{g})\right)\right) \\
& =\mathbf{C}_{\lambda+\rho} \otimes_{V_{\theta}}\left(\mathscr{U}(\mathfrak{g}) / \mathfrak{n}_{x} \mathscr{U}(\mathfrak{g})\right) /\left(\gamma\left(J_{\theta}\right)\left(\mathscr{U}(\mathfrak{g}) / \mathfrak{n}_{x} \mathscr{U}(\mathfrak{g})\right)\right) \\
& =\mathbf{C}_{\lambda+\rho} \otimes_{V_{\theta}}\left(\mathscr{U}(\mathfrak{g}) /\left(J_{\theta} \mathscr{U}(\mathfrak{g})+\mathfrak{n}_{x} \mathscr{U}(\mathfrak{g})\right)\right)=\mathbf{C}_{\lambda+\rho} \otimes_{V_{\theta}} H_{0}\left(\mathfrak{n}_{x}, \mathscr{U}_{\theta}\right) .
\end{aligned}
$$

5. Remark. Using spectral sequences instead of derived categories, 4 implies the following statement: The Grothendieck spectral sequences for composition of derived functors with $E_{2}$-terms $E_{2}^{p, q}=L^{p} T_{x}\left(L^{q} \Delta_{\lambda}(V)\right)$ and $E_{2}^{p, q}=$ $\operatorname{Tor}_{-p}^{V_{\theta}}\left(\mathbf{C}_{\lambda+\rho}, H_{-q}\left(\mathfrak{n}_{x}, V\right)\right)$ converge to the same limit.

To prove the theorem it is enough to establish the following fact. 
6. Lemma. Let $\lambda \in \mathfrak{h}^{*}$ be singular. Then there exists $V \in \mathscr{M}\left(\mathscr{U}_{\theta}\right)$ such that $L \Delta_{\lambda}(D(V))$ is not a cohomologically bounded complex.

Proof. Since the functor $T_{x}$ has finite left cohomological dimension, it is enough to find a $\mathscr{U}_{\theta}$-module $V$ such that $L T_{x}\left(L \Delta_{\lambda}(V)\right)$ is not a bounded complex for some $x \in X$. By 4, this is equivalent to the fact that $D\left(\mathbf{C}_{\lambda+\rho}\right) \stackrel{L}{\otimes}_{V_{\theta}}\left(D(\mathbf{C}) \stackrel{L}{\otimes}_{\mathscr{U}\left(\mathbf{n}_{x}\right)} D(V)\right)$ is not a cohomologically bounded complex.

Let $w_{0}$ be the longest element in $W$. Fix a Borel subalgebra $\mathfrak{b}_{0}$, and consider the Verma module $M\left(w_{0} \lambda\right)=\mathscr{U}(\mathfrak{g}) \otimes_{\mathscr{U}\left(\mathfrak{b}_{0}\right)} \mathbf{C}_{w_{0} \lambda-\rho}$. Pick $x$ so that $\mathfrak{b}_{x}$ is opposite to $\mathfrak{b}_{0}$. Then, by the Poincaré-Birkhoff-Witt theorem, $\boldsymbol{M}\left(w_{0} \lambda\right)$ is isomorphic to $\mathscr{U}\left(\mathfrak{n}_{x}\right) \otimes_{\mathbf{C}} \mathbf{C}_{w_{0} \lambda-\rho}$ as $\mathscr{U}\left(\mathfrak{n}_{x}\right)$-module. This implies, since $\mathfrak{b}_{x}$ is opposite to $\mathfrak{b}_{0}$ and corresponding specializations differ by $w_{0}$, that

$$
H_{0}\left(\mathfrak{n}_{x}, M\left(w_{0} \lambda\right)\right)=\mathbf{C}_{\lambda+\rho},
$$

and $H_{p}\left(\mathfrak{n}_{x}, M\left(w_{0} \lambda\right)\right)=0$ for $p \in \mathbf{N}$. Therefore,

$$
D(\mathbf{C}) \stackrel{L}{\otimes}_{\mathscr{U}\left(\mathfrak{n}_{x}\right)} D\left(M\left(w_{0} \lambda\right)\right)=D\left(\mathbf{C}_{\lambda+\rho}\right),
$$

and

$$
D\left(\mathbf{C}_{\lambda+\rho}\right) \stackrel{L}{\otimes_{V_{\theta}}}\left(D(\mathbf{C}) \stackrel{L}{\otimes}_{\mathscr{U}\left(\mathfrak{n}_{x}\right)} D\left(M\left(w_{0} \lambda\right)\right)\right)=D\left(\mathbf{C}_{\lambda+\rho}\right) \stackrel{L}{\otimes_{V_{\theta}}} D\left(\mathbf{C}_{\lambda+\rho}\right) .
$$

Clearly, we have

$$
H^{-p}\left(D\left(\mathbf{C}_{\lambda+\rho}\right) \stackrel{L}{\otimes_{V_{\theta}}} D\left(\mathbf{C}_{\lambda+\rho}\right)\right)=\operatorname{Tor}_{p}^{V_{\theta}}\left(\mathbf{C}_{\lambda+\rho}, \mathbf{C}_{\lambda+\rho}\right), \quad p \in \mathbf{Z}_{+} .
$$

On the other hand, we have

$$
\operatorname{Tor}_{p}^{V_{\theta}}\left(\mathbf{C}_{\lambda+\rho}, \mathbf{C}_{\lambda+\rho}\right)=\operatorname{Tor}_{p}^{R_{\lambda}}(\mathbf{C}, \mathbf{C}), \quad p \in \mathbf{Z}_{+} .
$$

Since $R_{\lambda}$ is not a regular local ring by 3 , its homological dimension is infinite $([5,17.3 .1])$ and $\operatorname{Tor}_{p}^{R_{\lambda}}(\mathbf{C}, \mathbf{C}) \neq 0$ for $p \in \mathbf{Z}_{+}([5,17.2 .11])$.

This completes the proof of the theorem.

\section{REFERENCES}

1. A. Beilinson and J. Bernstein, Localisation de g-modules, C.R. Acad. Sci. Paris, Ser. I 292 (1981), 15-18.

2. ___, A generalization of Casselman's submodule theorem, in "Representation theory of reductive groups," Birkhäuser, Boston, 1983, pp. 35-52.

3. N. Bourbaki, Algèbre commutative, Masson, Paris.

4. __ Groupes et algèbres de Lie, Masson, Paris.

5. A Grothendieck, Eléments de géométrie algébrique IV, Publ. I.H.E.S. No. 20 (1964).

6. H. Hecht, D. Miličić, W. Schmid, J. A. Wolf, Localization and standard modules for real semisimple Lie groups I: The duality theorem, Inventiones Math. 90 (1987), 297-332.

7. A. Joseph, J. T. Stafford, Modules of k-finite vectors over semi-simple Lie algebras, Proc. London Math. Soc. 49 (1984), 361-384.

8. D. Miličić, Localization and representation theory of reductive Lie groups," (mimeographed notes, to appear).

Department of Mathematics, University of Utah, Salt Lake City, Utah 84112 